\title{
FOLIL MAPUDUNGUN: ORGANIZACIÓN DE UN MÉTODO CON ENFOQUE COMUNICATIVO PARA LA ENSEÑANZA-
} APRENDIZAJE DE LA LENGUA MAPUCHE

Folil Mapudungun: organization basis of communicative method for mapuche language teaching and learning

\author{
Rodrigo Becerra Parra*
}

Resumen

Folil Mapudungun, fruto de doce años de producción y de sistematización de objetivos y contenidos, es un método comunicativo multidimensional de enseñanza-aprendizaje de la lengua mapuche, como segunda lengua (L2), orientado a jóvenes y adultos, en un contexto donde el mapudungun es lengua propia del territorio, lengua heredada y comunitaria. Hasta el momento, el método está integrado por ocho lecciones de estudio, desarrolladas y publicadas. En este artículo se presenta en detalle la organización de contenidos según sus tres ejes: transversal (basado en seis categorías, tanto funcionales como formales), longitudinal (de bases discursiva, cultural y metodológica) y local (también de base metodológica). Se concluye que la concurrencia de los ejes definidos permite superar la dicotomía entre contenidos y metodología, a la vez que favorece el desarrollo de las diversas competencias que actúan en la comunicación cotidiana.

Palabras clave: Mapudungun, L2, enfoque comunicativo, método multidimensional.

Abstract

Folil Mapudungun is a multidimensional method, based on a communicative approach, for teaching and learning Mapuche as a second language (L2). It was designed for adult learners in a context where Mapudungun is a "language specific to the territory", a heritage language and a community language. It is also the result of twelve years of research aiming to formulate objectives, and produce and systematize contents. At present, the method is constituted by eight lessons, developed and published in a textbook. This paper presents the method content organization according to three axes: transverse organization (based on six functional and formal categories), longitudinal organization (based on discursive, cultural and methodological principles), and local organization (based on methodological principles as well). It is claimed that the use of these three axes allows us to overcome the methodology-content dichotomy, and to favor the development of the various communicative competences used in everyday life.

Key words: Mapudungun, L2, communicative approach, multidimensional method.

\section{INTRODUCCIÓN}

El ámbito de las lenguas indígenas ha sido un campo de teorización, discusión y práctica especialmente fecundo para las Humanidades y las Ciencias Sociales durante las últimas tres décadas. Los factores más influyentes en este proceso han sido sociales y académicos: por una parte, los cambios políticos y las nuevas perspectivas de relación 
entre los pueblos originarios y los Estados nacionales; por otra, los cambios paradigmáticos y teóricos disciplinarios, desarrollados en un diálogo ineludible con los cambios sociales.

En concordancia, en el plano específicamente sociolingüístico se manifiesta un panorama efervescente y complejo. Así, junto con la constatación de procesos de desplazamiento y sustitución de las lenguas indígenas (Chiodi y Loncón, 1995; Catrileo, 2010), se observan procesos de promoción de las identidades étnicas, etnogénesis, reetnificación (Bengoa, 2007; Bartolomé, 2006; Boccara, 2002) y comunalización (Golluscio, 2006). Así, en el mundo mapuche, junto con la constatación de algunos territorios con una baja cantidad de hablantes de mapudungun, especialmente de hablantes jóvenes (Universidad Tecnológica Metropolitana y Corporación Nacional de Desarrollo Indígena, 2008; Gundermann et al, 2011), se observa una tendencia hacia la reivindicación y la recuperación lingüísticas en nuevos contextos. Por ejemplo, Wittig (2009) constata la adquisición lingüística del mapudungun en la juventud y en la adultez, procesos de transición de bilingüismo pasivo a activo, conformación de redes de uso de la lengua mapuche en el contexto urbano y apropiación de los contextos educacionales formales como herramientas de transmisión y adquisición de la lengua.

Este proceso, multidireccional y cambiante, demuestra un interés creciente por el futuro de las lenguas y por llevar a cabo acciones sociales que las reivindican. Asimismo, los movimientos sociopolíticos y sociolingüísticos han sido centrales en la constitución de un giro educativo, que ha pasado de los estudios descriptivos a los aplicados, favoreciendo las intervenciones tendientes a la revitalización lingüística y al reconocimiento de los derechos sociales. De este modo, en el plano académico, el campo de la enseñanza de lenguas ha reunido, entre otros, a educadores, psicólogos, lingüistas, antropólogos y sociólogos. Cada disciplina, desde sus propios desarrollos, se ha desplazado desde visiones técnicas, estructurales y, en algunos casos, biologicistas -imperantes hasta la década de 1960-, hacia perspectivas dinámicas, constructivistas y procesuales (con énfasis en las interacciones, los discursos, la heterogeneidad social, las identidades y las interpretaciones culturales) (por ejemplo, Da Silva, 2001; Golluscio, 2002; Johnson, 2008). Así, la enseñanza-aprendizaje de lenguas indígenas se conceptualiza hoy de manera dinámica y diversa, empujada a su vez por diferentes actores sociales, académicos y no académicos.

Como resultado, las sociedades han ido participando y co-construyendo los diseños de planes y materiales educativos como respuesta a sus problemáticas locales. Así, en Chile, las políticas de educación intercultural y educación intercultural bilingüe, que se iniciaron como políticas públicas orientadas a los pueblos indígenas (Huenchullán, 2007), se sumaron a un movimiento social y educativo que se venía forjando desde al menos la primera parte del siglo XX (Cañulef, 1998; Donoso, 2010). Con posterioridad a su confirmación legal en la década de 1990, las políticas interculturales han sido cuestionadas y comienzan a ser demandadas -aunque con cambios- por los propios pueblos indígenas (López, 2007; Red por los Derechos 
Educativos y Lingüísticos de los Pueblos Indígenas de Chile [Red DELPICH], 2011). Estas tensiones han sido relevadas por el mundo académico, toda vez que se viene constatando la dificultad para la participación real y para la conformación de un diálogo real con las prácticas y los saberes de los pueblos indígenas, así como una ilusión de participación, oculta tras la terminología oficial que se puede constituir en una nueva forma de colonialismo (Boccara, 2012; Pozo, 2014).

En particular, en el caso de la enseñanza de la lengua mapuche, el Ministerio de Educación y la Corporación Nacional de Desarrollo Indígena (CONADI) se han sumado a los actores independientes, académicos y no académicos, que ya venían actuando desde antes de los años noventa (por ejemplo, De Moesbach, 1963; Antileo, 1976; Alonqueo, 1987; Catrileo, 1987; Raguileo, 1990) en el desarrollo de iniciativas de enseñanza. En retrospectiva, se pueden mencionar tres actividades que han concitado interés y continuidad en el tiempo: i) la realización de cursos de lengua mapuche en distintas ciudades de Chile y Argentina; ii) la elaboración de material pedagógico (planes, textos y materiales didácticos complementarios) (Hasler et al, 2011; Norin et al, 2013); y, más recientemente, iii) la creación de programas educativos interculturales o bilingües (jardines infantiles y escuelas) (por ejemplo, Ministerio de Educación, 2011a, 2011b, 2013a, 2013b, entre otros).

Sin embargo, las iniciativas descritas no han logrado tener la aplicación, ni la generalidad, la influencia y los resultados esperados (Ministerio de Educación, 2011c; Red DELPICH, 2011; Treviño et al, 2012). Por estos motivos, surgen nuevas preguntas y desafíos que permiten cuestionar la idoneidad de los caminos trazados y de los planes delineados para lograr la revitalización de la lengua mapuche. En este contexto, el artículo presenta la fundamentación, resultados y discusión de un método de enseñanzaaprendizaje de la lengua mapuche denominado Folil Mapudungun. En primer lugar, se discuten los supuestos teóricos subyacentes en la elaboración del método. A continuación se describen las características de este, así como su organización y su etapa actual de desarrollo. Finalmente, se introduce una reflexión respecto de las características del programa del método en relación con sus inspiraciones y objetivos, considerando que forma parte de un proceso continuo de elaboración, revisión y readecuación para responder a los requerimientos de los procesos localizados de recuperación lingüística. En este sentido, la Lingüística, la Pedagogía y las Ciencias Sociales tienen un rol reflexivo y autorreflexivo de cara a las problemáticas sociales.

\section{FUNDAMENTACIÓN TEÓRICA}

\subsection{MÉTODOS DE ENSEÑANZA-APRENDIZAJE DE LENGUAS}

El fundamento teórico de todo método de enseñanza-aprendizaje de una segunda lengua (L2) corresponde a la serie de supuestos acerca de qué es el lenguaje y en qué consiste el proceso de enseñanza-aprendizaje de una lengua (Johnson, 2008). Anthony (1963) propuso una jerarquía de tres niveles, desde lo más general - de carácter teórico- 
a lo más específico - de carácter aplicado-, en la que diferencia entre enfoque, método y técnica. El primero es identificado como el nivel macro del proceso de enseñanzaaprendizaje, es decir, el fundamento teórico de carácter axiomático. El método, por su parte, correspondería a un plan de presentación sistemática del lenguaje, fundado en un enfoque particular. Por último, las técnicas corresponden al nivel de ejecución específico en las que se llevan a cabo actividades en la sala de clases, las que son coherentes con el método. Según Anthony (1963) "la técnica lleva a cabo un método que es consistente con un enfoque" (p. 63).

Sin embargo, a pesar de la influencia que ha ejercido la tripartición de Anthony, no existe un consenso generalizado acerca del alcance de los conceptos ni de posición en una jerarquía relativa. Por ejemplo, Richards y Rodgers (1982) denominaron enfoque, diseño y proceso a los conceptos propuestos por Anthony, redefiniendo "método" como la articulación de los tres, es decir, como un término amplio que consiste en la articulación de la teoría y la práctica.

Más allá de las divergencias conceptuales, en este trabajo definimos "enfoque" como el conjunto de supuestos, creencias y teorías acerca de la naturaleza del lenguaje y del proceso de enseñanza-aprendizaje de una lengua. A partir de esta serie de supuestos se conciben algunas "grandes ideas" que fundamentan la propuesta de un método (Johnson, 2008, p. 253): por ejemplo, "organización directa de la experiencia en términos lingüísticos", "favorecer la exposición de los estudiantes a textos reales y no controlados de lengua", o "las nociones y funciones constituirían la base de programas de estudio, en vez de un listado de estructuras gramaticales".

Mientras los conceptos de método y enfoque apuntan a la globalidad teóricometodológica de la propuesta educativa en la enseñanza de segundas lenguas, el concepto de "programa" tiene la utilidad de apuntar a la presentación formal de estas propuestas, lo que incluye de manera preferente la definición de contenidos y su organización. Si bien la definición de este término también es centro de discusión, al punto que se lo llega a utilizar como sinónimo de currículum (Johnson, 2008), en este artículo definimos programa como "la especificación y ordenación del contenido de uno o varios cursos" (García, 2000, p. 13). Según este último autor, el programa forma parte del currículum, concepto que define de manera polisémica, correspondiente tanto al plan de estudio (producto) como a los factores intervinientes en su formulación y puesta en práctica (proceso).

En las décadas de 1960 y 1970 diversas escuelas plantearon varias propuestas teóricas acerca de la naturaleza del lenguaje, y nuevas metodologías de estudio y aplicación. Se cuestionó la concepción del lenguaje como una facultad centrada en una competencia meramente lingüística, así como la posibilidad de obtener un real conocimiento de este y de su aprendizaje a partir de estudios del sistema gramatical en sí mismo, sin alusión a factores sociales ni contextuales. Esta apertura, denominada "revolución sociolingüística", ha consistido en la aparición de la sociolingüística, la etnografía de la comunicación, la teoría de los actos de habla, el análisis de la 
conversación, algunas escuelas funcionalistas y los estudios del discurso, entre otros acercamientos teórico-metodológicos (Duranti, 2003; Golluscio, 2002).

En este marco, a partir de la década de 1970 surge el enfoque comunicativo como una perspectiva para la enseñanza-aprendizaje de lenguas que parte de las necesidades comunicativas de los aprendices en situaciones comunicativas efectivas (Canale y Swain, 1980). Debido a que este enfoque concibe la interdependencia de la lengua y la comunicación, trabaja con el concepto amplio de "competencia comunicativa" (Hymes, 1972, p. 281), que incluye: i) la posibilidad formal de un enunciado (más o menos equivalente a la competencia lingüística chomskiana); ii) su viabilidad; iii) su calidad de apropiado (adecuado, feliz, exitoso) en un contexto específico; y iv) su uso efectivo en dicho contexto. Con posterioridad, a la luz de los desarrollos del análisis de la conversación y de las teorías de la acción comunicativa, Gumperz (2002) propone redefinir la competencia comunicativa como "el conocimiento de las convenciones lingüísticas y las convenciones comunicativas vinculadas a ellas que los hablantes deben poseer para iniciar y sostener el compromiso conversacional. El compromiso conversacional es, claramente, una precondición necesaria para la comprensión" (p. 154). Por tanto, en el enfoque comunicativo, la competencia comunicativa se sitúa en el centro de las decisiones que relacionan las necesidades de los estudiantes con la elaboración de programas, metodologías y técnicas.

Asimismo, desde el ámbito de la Lingüística aplicada a la enseñanza de lenguas, Canale y Swain (1980) definen la competencia comunicativa como la relación e interacción entre tres tipos de competencia: la competencia gramatical -conocimiento internalizado de reglas de buena formación de oraciones-, la competencia sociolingüística -las reglas de uso socioculturales y discursivas de la lengua-, y la competencia estratégica -las estrategias de comunicación, tanto verbales como no verbales-. De manera similar, el Consejo de Europa (2002) reconoce tres componentes de la competencia comunicativa: el lingüístico -relacionado a la lengua en tanto sistema-, el sociolingüístico -condiciones socioculturales del uso de la lengua- y el pragmático - uso de recursos lingüísticos según guiones interaccionales-.

De este modo, el enfoque comunicativo se liga a la Lingüística Funcional al basarse en una concepción del lenguaje como comportamiento, como acciones que se realizan con un fin y que tienen la capacidad de significar. En palabras de Halliday (2005), el lenguaje es "la codificación de un 'potencial de conducta' en un 'potencial de significado" (p. 33), es decir, un recurso en donde un hablante transforma lo que "puede hacer" en la interacción con otros individuos, en lo que "puede significar" al interior de su comunidad. En otras palabras, el lenguaje, al brindar significados compartidos por una cultura, es un medio en el que se manifiesta el potencial de acción de los hablantes, ya que ellos "significan" a través de un "hacer lingüístico".

En esta perspectiva se insiste en la importancia del contexto cultural y situacional, por lo que se deben tomar en cuenta las variables sociales en la planificación 
y ejecución de un método. Se considera que los significados son compartidos socialmente, y que se manifiestan e interpretan de forma contextual y en relación con situaciones significativas particulares, por lo que la enseñanza-aprendizaje de una L2 no puede aislar los significados de las intenciones comunicativas y de las funciones que vehiculiza. Además, porque el lenguaje es, según Halliday (2005), primaria y esencialmente una conducta social que se aprende y se explica mediante la interacción, aprender el lenguaje consiste en manejar las posibles acciones que con él se realizan.

Como resultado, el conocimiento de la lengua se concibe como una articulación de competencias que el estudiante debe manejar en la comunicación cotidiana. De esta forma, la competencia comunicativa tiene un carácter múltiple, de imbricación lingüística-cultural, y creativo, ya que trasciende el conocimiento de normas lingüísticas y de convenciones que se manifiestan en la comunicación, para incluir la capacidad del hablante de utilizar, adaptar e incluso modificar dichas normas y convenciones. Según las teorías funcionalistas y cognitivas corporizadas, tanto el conocimiento lingüístico como el aprendizaje están basados en el uso (Littlemore, 2011), ya que el conocimiento lingüístico está permanentemente sometido a actualización y modificación a partir de la experiencia. A causa de que el uso no está aislado del sistema social ni de los demás sistemas cognitivos, ni es meramente pasivo, sino que supone decisiones y elección de construcciones (por ende, construals) por parte de los hablantes, un enfoque comunicativo de enseñanza-aprendizaje de lenguas debe considerar la articulación de la lengua en la realidad social (situaciones comunicativas), funcional (intenciones, funciones comunicativas) y cognitiva (progresión y reflexividad del proceso de enseñanza-aprendizaje).

\subsection{MÉTODOS MULTIDIMENSIONALES}

Definimos un método como un plan de presentación sistemática de una lengua y, a la vez, como una articulación teórico-metodológica con vistas a plasmarse en un programa determinado, materiales de clase y actividades, que se establece a partir del macronivel teórico del enfoque. Es decir, constituye el sustrato y el esquema general para la preparación e implementación de la enseñanza-aprendizaje de una L2, en el que se expresa la teoría en la práctica. Brown (2001) agrega que un método es "un ordenamiento de especificaciones de clase para lograr objetivos lingüísticos. Los métodos tienden a relacionarse primariamente con los roles y los comportamientos de los profesores y alumnos, y secundariamente con los objetivos lingüísticos y de contenido, secuencias y materiales" (mi traducción, p. 15).

El primer método que se preparó con un enfoque comunicativo fue el nocionalfuncional. Este buscó resolver la separación entre conocimiento y práctica, visible en estudiantes gramaticalmente competentes, pero comunicativamente incompetentes. De esta forma, en la década de 1970 la organización conocida como Consejo de Europa tuvo la misión de fomentar la cooperación entre los países miembros, incluyendo la definición de un método para la enseñanza-aprendizaje de los idiomas europeos. Como 
resultado, Wilkins (1972) definió dos categorías a partir de las que se elaborarían programas orientados hacia la competencia comunicativa: las nociones y las funciones. Mientras las primeras se refieren a categorías de tipo semántico-gramatical (conceptuales), las segundas incluyen los usos de la lengua, haciendo alusión a los actos de habla.

Con posterioridad a la aparición del método nocional-funcional (o nociofuncional), la didáctica de lenguas se ha enriquecido con otros métodos que pretenden profundizar la práctica de la competencia comunicativa. A la vez que se ha criticado al método nociofuncional por continuar impartiendo contenidos formales bajo la apariencia de una organización funcional, han aparecido otros métodos: aquellos que hacen énfasis en el proceso de aprendizaje por sobre el producto (por ejemplo métodos procedimentales, basados en tareas, naturales) (Johnson, 2008) y aquellos que organizan un programa a partir de diversas categorías, haciendo recaer el foco del curso en una $u$ otra de ellas con la intención explícita de equilibrar la atención en el contenido y la metodología (método multidimensional).

En este panorama, los métodos multidimensionales organizan sus contenidos y su programa a base de una gama de categorías, y no solo a las nociones y las funciones. De hecho, "la base de un programa multidimensional es que tiene más de una unidad de organización" (Johnson, 2008, p. 360). De este modo, otras categorías usuales de organización son las estructuras (entendidas como "exponentes" de una función determinada), los temas (aquello de lo que se habla en una lección), las situaciones (que incluyen el entorno, las actividades y los roles, entre otros elementos) y la fonología (segmental y suprasegmental) (Tabla 1).

Tabla 1. Ejemplos de algunas categorías comunes de organización de un método multidimensional

\begin{tabular}{|c|l|}
\hline Categorías & \multicolumn{1}{|c|}{ Ejemplos } \\
\hline Nociones & Números, lugares, tiempo, ocupaciones, compañía, presente, futuro, etc. \\
\hline Funciones & Presentarse, preguntar, dar información, describir personas, invitar, etc. \\
\hline Estructuras & $\begin{array}{l}\text { Conjugaciones verbales, sufijo de futuro gramatical, patrón oracional ADJ + } \\
\text { NOM }^{1} \text {. }\end{array}$ \\
\hline Temas y situaciones & Presentaciones, familia, ocupaciones, comidas, gustos, etc. \\
\hline Fonología & Fonema/r/, acento léxico, entonación de preguntas, etc. \\
\hline
\end{tabular}

Mediante este esquema organizativo, los métodos mutidimensionales pretenden generar el aprendizaje de la lengua a base de las funciones que suele cumplir en relación con un tema, en una situación específica. Al mismo tiempo, se espera favorecer en el estudiante la capacidad de realizar generalizaciones importantes en y para el uso de un idioma, sin remitirse meramente al aprendizaje de unas cuantas frases útiles. Así, podrá

\footnotetext{
${ }^{1}$ Esta notación indica una construcción integrada por un adjetivo seguido de un nombre (sustantivo).
} 
desenvolverse en contextos culturalmente significativos, y transitar desde los usos más recurrentes y estereotipados a la creatividad en situaciones novedosas.

\section{FOLIL MAPUDUNGUN}

\subsection{CARACTERÍSTICAS GENERALES}

Folil Mapudungun se define como un método multidimensional con enfoque comunicativo de enseñanza-aprendizaje de la lengua mapuche, como segunda lengua (L2), orientado a adultos y jóvenes, en un contexto donde el mapudungun es lengua propia $\mathrm{y}$, en muchos casos, heredada y comunitaria ${ }^{2}$. Este método surgió de la constatación de la necesidad del aprendizaje de la lengua, expresada en la alta convocatoria que tuvieron los cursos de lengua mapuche desde que comenzaron a implementarse en 2002 en la Universidad de Concepción. Además, se constató la necesidad de un método con las características mencionadas, que privilegiara la comunicación efectiva en mapudungun y el desarrollo de competencias comunicativas de diversa índole. Es posible destacar, finalmente, que el método -y por tanto los materiales en que este se plasma- está orientado a apoyar procesos de enseñanzaaprendizaje guiados por un profesor hablante de la lengua mapuche. En consecuencia, el método presentado no responde a otros requerimientos de materiales educativos; por ejemplo, los autodidactas se encuentran aún insuficientemente satisfechos en el contexto de las lenguas indígenas en general, y de la lengua mapuche en particular. Sin embargo, este método provee cierta flexibilidad al respecto, favorece el descubrimiento del aprendiz y su estudio en solitario.

\subsection{ORGANIZACIÓN Y PRINCIPIOS ORGANIZATIVOS}

Folil Mapudungun es un método multidimensional de enseñanza-aprendizaje de la lengua mapuche, cuyos fundamentos y plan de organización se manifiestan en ocho lecciones de estudio (kimkülen, del mapudungun 'saber, estar consciente') desarrolladas y publicadas en formato de libro de texto (Norin et al, 2013). Además, se declara la planificación de ocho lecciones más, parcialmente desarrolladas, con las que conformarían el método: "Un plan de continuación de este texto por otro: la segunda entrega de Folil Mapudungun, también de 8 lecciones" (p. 5). Presenta tres ejes de organización:

1. Organización transversal: corresponde a una malla de categorías que organiza todas las lecciones del método.

\footnotetext{
${ }^{2}$ Se entiende como "lengua propia de un territorio" la lengua de una comunidad históricamente establecida en dicha región (Conferencia Mundial de los Derechos Lingüísticos, art. 1); y como lengua heredada o comunitaria, aquella que forma parte de la herencia familiar y cultural del individuo. Aunque los conceptos de lengua heredada y lengua comunitaria suelen confundirse, realizan énfasis distintos (Wiley, 2014). 
2. Organización longitudinal: realizada a partir de tres principios organizativos, a partir de estos se presenta un ordenamiento del método en bloques secuenciales.

3. Organización local: corresponde a una secuencia de organización progresiva de los contenidos y actividades en cada lección.

\subsubsection{ORGANIZACIÓN TRANSVERSAL}

En tanto método multidimensional, Folil Mapudungun sistematiza y presenta su plan de contenidos y actividades a partir de distintas categorías de organización paralela: i) funciones y destrezas; ii) temas y situaciones; iii) nociones; iv) léxico; v) estructuras; y vi) fonología. Estas categorías organizan el método de manera transversal, por lo que cada una de las ocho lecciones desarrolladas se basa en ellas.

Estas seis categorías expresan el análisis de necesidades y la definición de objetivos llevados a cabo durante la construcción del programa del método (García, 2000; Zabalza, 2009) ${ }^{3}$. Así, las tres primeras categorías tienen una orientación funcional (uso del conocimiento, en este caso, de la lengua), mientras que las últimas tres se orientan hacia la forma (familiarización con los elementos y las construcciones lingüísticas, particularmente con la de naturaleza léxico-gramática y el sistema fonológico). Por este motivo, la categoría 2 ("funciones y destrezas") corresponde a los "objetivos comunicativos" del método, los que permiten hablar en las situaciones reconocidas en la categoría 1 ("temas y situaciones") y sobre los temas expresados en la categoría 3 ("nociones"); a la vez que las categorías 5, 6 y 7 ("léxico", "estructuras" y "fonología") corresponden a los "objetivos lingüísticos" del método, que permiten desarrollar las funciones declaradas. Como se indicó en el marco teórico, en el lenguaje no existe una separación entre forma y función, por lo que la definición de ambos tipos de objetivos se realizó de manera dialéctica y contextualizada. De no ser así, la planificación de objetivos comunicativos desligados de los objetivos lingüísticos, o viceversa, conduciría a un falseamiento de las expresiones de la lengua -al no considerar las formas efectivamente utilizadas- o a una desnaturalización de la gramática y del léxico -desvinculados de su uso-.

La organización basada en las seis categorías señaladas permite presentar de manera holística los aspectos socioculturales, las acciones desarrolladas en y con dichos contextos, y los elementos lingüísticos que se espera maneje el aprendiz de manera progresiva. A continuación se presenta la organización de la primera lección (Tabla 2), con una glosa (en la primera fila) para leer la interrelación práctica entre las seis categorías.

\footnotetext{
${ }^{3}$ La exposición detallada de la construcción del programa, así como la discusión acerca de los factores intervinientes y las tensiones de relevancia pedagógica y sociocultural, exceden el alcance de este artículo, por lo que serán desarrolladas en otro trabajo.
} 


\section{Rodrigo Becerra Parra}

Tabla 2. Resumen de la organización de la primera lección del método Folil Mapudungun, con glosa explicativa para la lectura de la interrelación de las categorías

\begin{tabular}{|c|c|c|c|c|c|c|}
\hline Lección & $\begin{array}{c}\text { Temas y } \\
\text { situaciones }\end{array}$ & $\begin{array}{c}\text { Funciones y } \\
\text { destrezas }\end{array}$ & Nociones & Léxico & Estructuras & Fonología \\
\hline & $\begin{array}{l}\text { En las siguientes } \\
\text { situaciones } \\
\text { comunicativas } \\
\text {... }\end{array}$ & $\begin{array}{l}\text {...los estudiantes } \\
\text { aprenderán a... }\end{array}$ & $\begin{array}{l}\text {...hablar } \\
\text { sobre... }\end{array}$ & $\begin{array}{l}\ldots \text {..con las } \\
\text { siguientes } \\
\text { palabras } \\
\ldots\end{array}$ & $\begin{array}{l}\text {...que manifiestan } \\
\text { las siguientes } \\
\text { formas y } \\
\text { construcciones... }\end{array}$ & $\begin{array}{l}\text {...y que } \\
\text { presentan la } \\
\text { siguiente } \\
\text { pronunciación }\end{array}$ \\
\hline 1 & $\begin{array}{l}\text { Saludos, } \\
\text { despedidas, } \\
\text { conocimiento } \\
\text { mutuo }\end{array}$ & $\begin{array}{l}\text { Saludary } \\
\text { despedirse, } \\
\text { informar si se } \\
\text { está bien o mal, } \\
\text { nombrey } \\
\text { procedencia }\end{array}$ & $\begin{array}{l}\text { Estado de } \\
\text { salud, } \\
\text { nombre, } \\
\text { proceden- } \\
\text { cia, } \\
\text { identidad }\end{array}$ & $\begin{array}{l}\text { Formas } \\
\text { de saludo } \\
\text { y } \\
\text { tratamien- } \\
\text { to, verbos } \\
\text { de } \\
\text { proceden- } \\
\text { cia y } \\
\text { residencia }\end{array}$ & $\begin{array}{l}\text { Pronombres y } \\
\text { conjugaciones de } \\
1^{\mathrm{a}}, 2^{\mathrm{a}} \mathrm{y} 3^{\mathrm{a}} \text { persona } \\
\text { singulares; } \\
\text { oraciones } \\
\text { nominales; } \\
\text { oraciones } \\
\text { negativas; } \\
\text { partículas } p u \mathrm{y} \\
\text { mew }\end{array}$ & $\begin{array}{l}\text { Sonido del } \\
\text { fonema } \\
\text { expresado por } \\
\text { la grafia “' } r \text { ", } \\
\text { entonación } \\
\text { interrogativa y } \\
\text { afirmativa }\end{array}$ \\
\hline
\end{tabular}

\subsubsection{ORGANIZACIÓN LONGITUDINAL}

Folil Mapudungun está organizado de manera longitudinal a base de los siguientes criterios: i) discursivo; ii) cultural; y iii) metodológico, con la finalidad de enfatizar y favorecer la conexión de los contenidos con su aplicación.

\subsubsection{CRITERIO DISCURSIVO: PRINCIPIO DE RELACIÓN ENTRE TEXTOS, SITUACIONES Y SIGNIFICADOS}

En relación con el primero de estos criterios, el plan del método se organiza en partes denominadas troy (del mapudungun, 'articulación, nudillo, segmento'), integradas por cuatro lecciones cada una. De este modo, la planificación hasta aquí publicada consta de dos troy, y las lecciones planificadas constituirían dos más. Con el fin de familiarizar al aprendiz con distintas estrategias discursivas socioculturalmente pertinentes, y que tiendan a una cobertura progresiva de las estrategias existentes en el discurso mapuche, cada troy está destinada a un tipo de secuencia textual (Adam, 1992; Calsamiglia y Tusón, 2007). La primera se dedica a la práctica de las secuencias dialogales y descriptivas, en tanto que la segunda se orienta a la familiarización de las secuencias dialogales y explicativas ${ }^{5}$.

Las secuencias dialogales son típicamente poligestionadas, a diferencia de las descriptivas, explicativas, argumentativas y narrativas, que son prototípicamente

\footnotetext{
${ }^{4}$ En las Tablas 3 y 4 se abrevia la explicación de la categoría “fonología”, por motivos de espacio y sencillez para los aprendices.

${ }^{5}$ De manera equivalente, el método Folil Mapudungun declara que la segunda entrega, compuesta por las partes (troy) 3 y 4, está orientada, respectivamente, a la familiarización con las secuencias textuales argumentativas y narrativas. 
(aunque no única ni necesariamente) monogestionadas. Las secuencias descriptivas se caracterizan por presentar la realidad deteniéndose en sus propiedades, partes y relaciones, a partir de la percepción directa del sujeto hablante y de su subjetividad, y considerando la contextualización de su discurso (Calsamiglia y Tusón, 2007). Además, estas secuencias se elaboran a partir de un anclaje textual, que presenta el tema o referente, después de ello se manifiestan las estrategias descriptivas: enumeración, jerarquización, comparación, presentación o autopresentación, entre otras. Por otro lado, las secuencias explicativas se caracterizan por presuponer la existencia de información, la que se orientan a clarificar y complementar ("hacer saber"), mediante diversas estrategias: definición, reformulación, ejemplificación y citación, entre otras. Frecuentemente las explicaciones responden a una pregunta, implícita o explícita, acerca del porqué de algún asunto (Adam, 1992; Calsamiglia y Tusón, 2007).

Frente a la diversidad y amplitud de las estrategias explicativas, la parte (troy) 2 enfatiza el dominio de los actos de habla representativos ${ }^{6}$, expresivos y algunos directivos (sensu Searle, 1975). Los actos representativos o asertivos tienen como propósito representar un estado de cosas como real; en otras palabras, hacer saber cómo es o puede ser la realidad (expresar certeza o posibilidad, o bien negarlas). Por su parte, los actos expresivos buscan manifestar el estado psicológico o las actitudes del hablante respecto de lo dicho y al estado de cosas (incluyendo a sí mismo y al oyente): por ejemplo, expresar agradecimiento o disculpas, expresar gusto y deseo, expresar sentimientos. Por último, los actos directivos buscan lograr que el oyente realice algo: por ejemplo, preguntar, pedir, aconsejar, invitar, advertir, etc. De esta manera se busca transitar desde la conversación centrada en la descripción de hechos, al significado que ellos tienen para los interlocutores y para la interacción.

Como resultado, las cuatro primeras lecciones se caracterizan por tratar principalmente situaciones dialógicas y de descripción de sí mismo, de las personas o de la situación, mientras que las cuatro lecciones siguientes introducen, junto con la comunicación dialógica - que sigue siendo foco prioritario de estudio-, los actos de explicación ligados a las disposiciones y actitudes del hablante (clarificaciones, deseos, preferencias, consejos) (Tablas 3 y 4 ).

Tabla 3. Malla de categorías de organización transversal del método multidimensional Folil Mapudungun, parte 1

\begin{tabular}{|c|c|c|c|c|c|c|}
\hline & $\begin{array}{c}\text { Temas y } \\
\text { situaciones }\end{array}$ & $\begin{array}{l}\text { Funciones y } \\
\text { destrezas }\end{array}$ & Nociones & Léxico & Estructuras & Fonología \\
\hline 1 & $\begin{array}{l}\text { Saludos, } \\
\text { despedidas, } \\
\text { conocimient } \\
\text { o mutuo }\end{array}$ & $\begin{array}{l}\text { Saludary } \\
\text { despedirse, } \\
\text { informar si se } \\
\text { está bien o } \\
\text { mal, nombre y } \\
\text { procedencia }\end{array}$ & $\begin{array}{l}\text { Estado de } \\
\text { salud, nombre, } \\
\text { procedencia, } \\
\text { identidad }\end{array}$ & $\begin{array}{l}\text { Formas de } \\
\text { saludo y } \\
\text { tratamiento, } \\
\text { verbos de } \\
\text { procedenciay } \\
\text { residencia }\end{array}$ & $\begin{array}{l}\text { Pronombres y } \\
\text { conjugaciones de } 1^{\mathrm{a}}, 2^{\mathrm{a}} \\
\text { y } 3^{\mathrm{a}} \text { persona singulares; } \\
\text { oraciones nominales; } \\
\text { oraciones negativas; } \\
\text { partículas pu y mew }\end{array}$ & $\begin{array}{l}\text { Sonido "r", } \\
\text { entonación } \\
\text { interrogati- } \\
\text { va y } \\
\text { afirmativa }\end{array}$ \\
\hline
\end{tabular}

\footnotetext{
${ }^{6}$ También denominados "asertivos".
} 


\begin{tabular}{|c|c|c|c|c|c|c|}
\hline 2 & $\begin{array}{l}\text { Presentación } \\
\text { personal }\end{array}$ & $\begin{array}{l}\text { Complementa } \\
\text { r la } \\
\text { presentación } \\
\text { personal } \\
\text { (edad, familia, } \\
\text { pareja, } \\
\text { ocupación), } \\
\text { hablar de la } \\
\text { familia y de } \\
\text { otras personas }\end{array}$ & $\begin{array}{l}\text { Küpalme } \\
\text { ('procedencia } \\
\text { familar'), } \\
\text { números, } \\
\text { edad, estado } \\
\text { civil }\end{array}$ & $\begin{array}{l}\text { Parentesco } \\
\text { cercano, } \\
\text { números, } \\
\text { ocupaciones, } \\
\text { estado civil }\end{array}$ & $\begin{array}{l}\text { Pronombres y } \\
\text { conjugaciones de } 3^{\text {a }} \\
\text { persona dual y plural; } \\
\text { posesivos; frases } \\
\text { posesivas; interrogativos } \\
\text { iney y chum-; morfema } \\
\text {-nge }\end{array}$ & $\begin{array}{l}\text { Sonidos } \\
\text { "ng”y “"ñ”, } \\
\text { cambio de } \\
\text { posición del } \\
\text { acento } \\
\text { léxico }\end{array}$ \\
\hline 3 & $\begin{array}{l}\text { Reunión y } \\
\text { presentación } \\
\text { de personas } \\
\text { y entorno }\end{array}$ & $\begin{array}{l}\text { Presentar a } \\
\text { alguien, hablar } \\
\text { del tiempo } \\
\text { atmosférico, } \\
\text { de las } \\
\text { sensaciones } \\
\text { (sed, calor, } \\
\text { etc.), de la } \\
\text { reunión y el } \\
\text { lugar }\end{array}$ & $\begin{array}{l}\text { Compañía, } \\
\text { reuniones, } \\
\text { tiempo } \\
\text { atmosférico, } \\
\text { fechas, } \\
\text { sensaciones }\end{array}$ & $\begin{array}{l}\text { Tiempo } \\
\text { atmosférico, } \\
\text { épocas o } \\
\text { estaciones del } \\
\text { año, } \\
\text { sensaciones }\end{array}$ & $\begin{array}{l}\text { Conjugación verbal } \\
\text { completa; pronombres } \\
\text { grupalizadores; } \\
\text { demostrativos; verbos } \\
\text { existenciales; morfemas } \\
\text {-nge,-le, } \\
-k e\end{array}$ & $\begin{array}{l}\text { Sonidos } \\
\text { "ü', } \\
\text { afirmaciones } \\
\text { enfáticas }\end{array}$ \\
\hline 4 & $\begin{array}{l}\text { Visitarse y } \\
\text { encontrarse } \\
\text { fortuitament } \\
\text { e }\end{array}$ & $\begin{array}{l}\text { Hablar de un } \\
\text { encuentro, de } \\
\text { una visita, de } \\
\text { la fecha; hacer } \\
\text { halagos y } \\
\text { comentarios } \\
\text { positivos sobre } \\
\text { algo }\end{array}$ & $\begin{array}{l}\text { Visita, } \\
\text { desplazarse, } \\
\text { momentos del } \\
\text { día }\end{array}$ & $\begin{array}{l}\text { Encuentros, } \\
\text { verbos de } \\
\text { desplazamient } \\
\text { o, momentos } \\
\text { del día }\end{array}$ & $\begin{array}{l}\text { Forma básica del verbo; } \\
\text { morfemas -pe y-tu; } \\
\text { posesivos duales y } \\
\text { plurales; interrogativos } \\
\text { chew y chumül; } \\
\text { morfema } \\
\text {-wen }\end{array}$ & $\begin{array}{l}\text { Sonido “ll", } \\
\text { entonación } \\
\text { en halagos }\end{array}$ \\
\hline
\end{tabular}

Tabla 4. Malla de categorías de organización transversal del método multidimensional Folil Mapudungun, parte 2

\begin{tabular}{|c|c|c|c|c|c|c|}
\hline & $\begin{array}{c}\text { Temas y } \\
\text { situaciones }\end{array}$ & $\begin{array}{c}\text { Funciones y } \\
\text { destrezas }\end{array}$ & Nociones & Léxico & Estructuras & Fonología \\
\hline 5 & $\begin{array}{l}\text { Cualidades } \\
\text { personales }\end{array}$ & $\begin{array}{l}\text { Describir } \\
\text { personas, } \\
\text { expresar } \\
\text { gustos, hablar } \\
\text { de las } \\
\text { actividades } \\
\text { habituales y } \\
\text { de su } \\
\text { frecuencia }\end{array}$ & $\begin{array}{l}\text { Cualidades, } \\
\text { colores, } \\
\text { gustos, } \\
\text { recreación }\end{array}$ & $\begin{array}{l}\text { Rasgos } \\
\text { personales, } \\
\text { colores, } \\
\text { actividades }\end{array}$ & $\begin{array}{l}\text { Cualidades con } \\
\text { verbos con - } \\
\text { nge y -le/küle, } \\
\text { o con } \\
\text { oraciones } \\
\text { nominales; } \\
\text { palabras de } \\
\text { frecuencia; } \\
\text { construcciones } \\
\text { adjetivo + } \\
\text { sustantivo; } \\
\text { ayüken + } \\
\text { sustantivo, } \\
\text { ayüken + tañi } \\
\text { + verbo + -ael, } \\
\text { y küpa + verbo }\end{array}$ & $\begin{array}{l}\text { Repaso de los } \\
\text { sonidos "r", } \\
\text { "ng" y "ü", } \\
\text { entonación de } \\
\text { enumeracione } \\
\text { s }\end{array}$ \\
\hline
\end{tabular}


Folil Mapudungun: organización de un método con enfoque comunicativo

\begin{tabular}{|c|c|c|c|c|c|c|}
\hline 6 & Invitación & $\begin{array}{l}\text { Realizar } \\
\text { invitaciones y } \\
\text { llegar a } \\
\text { acuerdo, } \\
\text { aceptar o } \\
\text { rechazar } \\
\text { invitaciones y } \\
\text { explicar las } \\
\text { razones, } \\
\text { indicar } \\
\text { direcciones }\end{array}$ & $\begin{array}{l}\text { Momentos } \\
\text { del día. } \\
\text { Invitaciones. } \\
\text { Direcciones }\end{array}$ & $\begin{array}{l}\text { Lugares de la } \\
\text { ciudad, horas, } \\
\text { direcciones y } \\
\text { posiciones (al } \\
\text { lado, al frente, } \\
\text { atrás, derecho, } \\
\text { etc.) }\end{array}$ & $\begin{array}{l}\text { Morfema-a de } \\
\text { futuro; } \\
\text { imperativo; } \\
\text { negación del } \\
\text { imperativo } \\
\text { (morfemas -kil } \\
\text { y-kinol); } \\
\text { construcción } \\
\text { pepi + verbo; } \\
\text { contraste entre } \\
\text { mew y püle; } \\
\text { fey como } \\
\text { conector } \\
\text { temporal }\end{array}$ & $\begin{array}{l}\text { Sonidos } \\
\text { interdentales } \\
\text { "nh" y "lh", } \\
\text { entonación de } \\
\text { las } \\
\text { invitaciones } \\
\text { con } \\
\text { imperativos }\end{array}$ \\
\hline 7 & $\begin{array}{l}\text { Comida y } \\
\text { recepción en } \\
\text { una casa. } \\
\text { Comida en un } \\
\text { restaurante }\end{array}$ & $\begin{array}{l}\text { Recibir a una } \\
\text { visita en casa, } \\
\text { saludar al } \\
\text { llegar, invitar } \\
\text { a pasar, } \\
\text { ofrecer, } \\
\text { aceptar, pedir } \\
\text { y rechazar } \\
\text { alimentos, } \\
\text { expresar } \\
\text { gustos y } \\
\text { deseos }\end{array}$ & $\begin{array}{l}\text { Comidas, } \\
\text { tipos de } \\
\text { invitaciones } \\
\text { (münatu, } \\
\text { konchotun, } \\
\text { kolle, misha, } \\
\text { chafkin, etc.). } \\
\text { Cantidad }\end{array}$ & $\begin{array}{l}\text { Alimentos, tipos } \\
\text { de alimentos, } \\
\text { porciones de } \\
\text { alimentos }\end{array}$ & $\begin{array}{l}\text { Verbalización } \\
\text { de alimentos } \\
\text { con -tu; } \\
\text { morfema -afu } \\
\text { en expresiones } \\
\text { corteses; } \\
\text { cuantificación } \\
\text { (rume, doy, } \\
\text { etc.); } \\
\text { construcciones } \\
\text { verbo + -lu + } \\
\text { trokifi-, y küpa } \\
\text { + verbo; } \\
\text { interrogativo } \\
\text { chuchi }\end{array}$ & $\begin{array}{l}\text { Sonidos "ch" } \\
\text { y "tr", } \\
\text { entonación de } \\
\text { los } \\
\text { ofrecimientos }\end{array}$ \\
\hline 8 & Enfermedades & $\begin{array}{l}\text { Hablar de } \\
\text { dolencias, } \\
\text { informar } \\
\text { acerca del } \\
\text { origen de las } \\
\text { dolencias, } \\
\text { indicar } \\
\text { frecuencia, } \\
\text { recomendar } \\
\text { un remedio, } \\
\text { pedir y dar } \\
\text { opiniones }\end{array}$ & $\begin{array}{l}\text { Enfermedad. } \\
\text { Problemas } \\
\text { Cuerpo } \\
\text { humano } \\
\text { Frecuencia }\end{array}$ & $\begin{array}{l}\text { Partes del } \\
\text { cuerpo, } \\
\text { dolencias y } \\
\text { enfermedades }\end{array}$ & $\begin{array}{l}\text { Verbo kutran-; } \\
\text { verbos pin y } \\
\text { fele-; conector } \\
\text { feymew; } \\
\text { construcción } \\
\text { müley + } \\
\text { posesivo + } \\
\text { verbo + -ael; } \\
\text { conjugaciones } \\
\text { agente- } \\
\text { paciente; } \\
\text { interrogativos } \\
\text { chumte, } \\
\text { chumten y } \\
\text { chumteñma }\end{array}$ & $\begin{array}{l}\text { Sonidos “g”y } \\
\text { "w", } \\
\text { entonación de } \\
\text { las } \\
\text { recomenda- } \\
\text { ciones }\end{array}$ \\
\hline
\end{tabular}




\subsubsection{CRITERIO CULTURAL: PRINCIPIO DE CONTEXTUALIZACIÓN Y DE RELACIÓN LENGUA-CULTURA}

Con relación al segundo criterio de organización longitudinal, el programa del método incluye secciones especialmente dedicadas a la profundización de ciertos aspectos de la cultura mapuche. Estas secciones, denominadas Ad Mapu (del mapudungun, 'características y aspectos del territorio' o 'cultura del territorio'), se disponen cada una o dos lecciones (Tabla 5), sumándose al tratamiento ineludible de la cultura durante el abordaje de los contenidos, así como a las secciones especialmente dedicadas a ella en cada lección (vid. infra " "Criterio metodológico").

Tabla 5. Secciones dedicadas a la profundización de los contenidos (en gris), dispuestas en relación con las partes y a las lecciones del método

\begin{tabular}{|c|c|c|}
\hline Parte & Lección & Nombre de la lección \\
\hline \multirow{6}{*}{$\begin{array}{l}\text { 1. Secuencias } \\
\text { textuales } \\
\text { dialógicas y } \\
\text { descriptivas }\end{array}$} & 1 & Kimuwün dungu ('Palabras/temas de conocimiento mutuo') \\
\hline & 2 & Tañi ineyngen ('Mi persona, quién soy'). \\
\hline & \multicolumn{2}{|r|}{ Ad Mapu: Tati pu mapuche } \\
\hline & 3 & Kom iñchiñ ('Todos nosotros'). \\
\hline & \multicolumn{2}{|r|}{ Ad Mapu: Wall Mapu } \\
\hline & 4 & Kom küme amuley! (‘¡Todo va bien!') \\
\hline & \multicolumn{2}{|r|}{ Ad Mapu: Tati рu reñma } \\
\hline \multirow{5}{*}{$\begin{array}{l}\text { 2. Secuencias } \\
\text { textuales } \\
\text { dialógicas y } \\
\text { explicativas }\end{array}$} & 5 & Femngeiñ ('Así somos') \\
\hline & 6 & Aтиуи may! ('¡Vamos los/las dos!') \\
\hline & \multicolumn{2}{|r|}{ Ad Mapu: Chumngen tati waria } \\
\hline & 7 & Matetuafuymi am? (‘'Tomarías mate?’) \\
\hline & 8 & Küme felen ('Estar bien') \\
\hline & \multicolumn{2}{|r|}{ Ad Mapu: Fünh anümka tami ikeek lelfün mew } \\
\hline
\end{tabular}

\subsubsection{CRITERIO METODOLÓGICO: PRINCIPIO DE ORIENTACIÓN A LA PRÁCTICA}

Respecto del tercer criterio organizativo longitudinal, el programa del método incluye, cada dos lecciones, secciones especiales con actividades de aprendizaje, que se suman a las actividades regulares dispuestas en el programa de todas las lecciones. Además, con el fin de favorecer el ingreso al uso de la lengua, este énfasis se refuerza en la primera parte del programa, mediante la densificación de secciones orientadas a las actividades de aprendizaje: un capítulo introductorio con los saludos, presentación de palabras y frases útiles, y una sección de ejercitación adicional después de la primera lección (Tabla 6). 
Tabla 6. Secciones dedicadas a la profundización de los contenidos (en gris), dispuestas en relación con las partes y a las lecciones del método

\begin{tabular}{|c|c|c|}
\hline Parte & Lección & Nombre de la lección \\
\hline \multirow{7}{*}{$\begin{array}{l}\text { 1. Secuencias } \\
\text { textuales } \\
\text { dialógicas y } \\
\text { descriptivas }\end{array}$} & Introducción & Chaliwaiñ! ('¡Saludémonos!') \\
\hline & 1 & $\begin{array}{l}\text { Kimuwün dungu ('Palabras/temas de conocimiento } \\
\text { mutuo') }\end{array}$ \\
\hline & \multicolumn{2}{|r|}{ Kake nütramkawün } \\
\hline & 2 & Tañi ineyngen ('Mi persona, quién soy'). \\
\hline & \multicolumn{2}{|r|}{ Küdawküdawtun } \\
\hline & 3 & Kom iñchiñ ('Todos nosotros'). \\
\hline & 4 & Kom küme amuley! (‘¡Todo va bien!’) \\
\hline \multirow{7}{*}{$\begin{array}{l}\text { 2. Secuencias } \\
\text { textuales } \\
\text { dialógicas y } \\
\text { explicativas }\end{array}$} & \multicolumn{2}{|r|}{ Küdawküdawtun } \\
\hline & 5 & Femngeiñ (‘Así somos’) \\
\hline & 6 & Атиуи may! ('¡Vamos los/las dos!') \\
\hline & \multicolumn{2}{|r|}{ Küdawküdawtun } \\
\hline & 7 & Matetuafuymi am? (‘'Tomarías mate?’) \\
\hline & 8 & Küme felen ('Estar bien') \\
\hline & \multicolumn{2}{|r|}{ Küdawküdawtun } \\
\hline
\end{tabular}

\subsubsection{ORGANIZACIÓN LOCAL}

Corresponde a la organización interna de cada lección, donde cada una de ellas está ordenada basada en dos criterios estructurales: i) funcional (criterios funcionales para la secuenciación de los contenidos y de las actividades); y ii) formal (calidad y cantidad de secciones integrantes, disposición de las secciones y número de páginas en el texto, de acuerdo con Norin et al., 2013).

\subsubsection{CRITERIO FUNCIONAL}

Al interior de cada lección, los contenidos se organizan de manera funcional, esto es, siguiendo criterios que apunten a una vinculación práctica entre ellos y los sujetos que participan de la relación pedagógica (profesor/a y estudiantes). Esto obedece a la necesidad de que todo método, en tanto plan de presentación sistemática del lenguaje, se nutra de los hallazgos de diversas investigaciones acerca de la naturaleza del lenguaje en general, de las características de la lengua de enseñanza particular, y de las experiencias de enseñanza-aprendizaje en contextos similares. Por tanto, en consonancia con el saber aplicado actual, la presentación de los contenidos se realiza a partir de actividades de aprendizaje en las que se utiliza la lengua de manera estratégica para resolver problemas, es decir, siguiendo una orientación a la acción (Consejo de Europa, 2002).

Así, la selección y secuenciación de contenidos está ineludiblemente ligada y retroalimentada por la selección y secuenciación de objetivos y de actividades. En este sentido, la estructuración funcional es necesaria para integrar los objetivos y los 
contenidos en el proceso didáctico del proyecto curricular (Zabalza, 2009). En especial, García (2000) propone considerar el potencial comunicativo y cognitivo de los ejercicios, para ello presenta dos escalas cualitativas. Según la primera de ellas, que considera el potencial comunicativo de las actividades, estas pueden gradarse entre aquellas que supongan menos y aquellas que supongan más interacción: es decir, desde la mera recepción de información; siguiendo por la vinculación formal del estudiante con la información; la transferencia de ella; la respuesta a dicha información; su aplicación; $\mathrm{y}$, finalmente, su negociación en contextos interactivos. Según la escala del potencial cognitivo, las actividades se gradarían entre las que suponen solo el conocimiento y memorización de información, pasando progresivamente por las que implican traslación, comprensión, aplicación, análisis y síntesis, hasta llegar a aquellas que implican su evaluación. Siguiendo estos criterios, Folil Mapudungun construye una progresión de las actividades, desde la recepción y vinculación formal con casos particulares de lengua, pasando por su análisis, generalización y aplicación práctica, hasta llegar a su uso e integración (para el detalle de la disposición de estas actividades, vid. infra, "Criterio formal").

\subsubsection{CRITERIO FORMAL}

Las lecciones tienen una extensión y un peso didáctico similares. De manera formal, en el material publicado (Norin et al., 2013), cada lección se extiende a lo largo de doce páginas, que distribuyen los contenidos en cinco secciones, en el mismo orden, y con la misma extensión. Como resultado, el texto es presentado siguiendo estricta regularidad y simetría. Además, cada sección se condice con la secuencialidad derivada del principio funcional recién señalado (vid. supra, 3.2.3, "Criterio funcional"), por lo que se produce una confluencia de metodología y contenidos, proceso y producto.

A continuación se presentan las cinco secciones de cada lección, así como sus contenidos y objetivos didácticos:

1. Wüneluchi dungu ('Primeras palabras o temas'): se ubica en la primera página de cada lección. Incluye el nombre de la lección, una foto de contextualización, una presentación de los objetivos comunicativos y lingüísticos de la unidad, una contextualización cultural y una canción relacionada al tema de la lección. Su objetivo es introducir al estudiante en el tema, promover sus inferencias y su anticipación cognitiva, con el fin de favorecer la comprensión y el aprendizaje (Figura 1).

2. Weke dungu ('Palabras o temas nuevos'): se presenta en las tres páginas siguientes. Incluye textos sencillos, frecuentemente dialógicos, y ejercicios variados, organizados en torno a situaciones y a tareas comunicativas. Cada vez que es posible, se proveen actividades que puedan ser desarrolladas a partir del descubrimiento de los estudiantes. Su objetivo es familiarizar a los aprendices con los objetivos comunicativos y lingüísticos de la lección, promover que ellos 
desarrollen las habilidades de comprensión e inferencia en la lengua, releven datos, generalicen el funcionamiento de la lengua, e investiguen contenidos temáticos que favorezcan la subsecuente consolidación de sus conocimientos.

3. Kimürpuam dungu ('Palabras o temas para conocer'): se subdivide en dos subsecciones: Chumngechi dungual ('Cómo hablar') y Fillke nemül ('Todas las palabras'), que se disponen en las tres páginas siguientes (dos páginas y una página, respectivamente). En la subsección Chumngechi dungual se resumen los aspectos gramaticales, en particular, las clases de palabras, las construcciones morfológicas y sintácticas. Por su parte, en la subsección Fillke nemül se presenta un resumen del léxico de la lección y de las expresiones más comunes. En ambas subsecciones se presentan ejercicios "que buscan que el estudiante no solo adquiera una competencia pasiva, sino que se enfrente a resolver problemas variados de forma imaginativa" (Norin et al., 2013, p. 8).

4. Nütramkayal ('Para conversar'): se presenta en las tres páginas siguientes. Incluye textos y actividades variadas, dispuestas a base de situaciones comunicativas. En consonancia con el criterio funcional expuesto, estas actividades enfatizan la aplicación de los contenidos mediante el enfrentamiento del estudiante a actividades de producción. Su objetivo es la puesta en práctica de las expresiones y del léxico aprendidos, y de las reglas lingüísticas estudiadas, mediante tareas enfocadas comunicativamente. Adicionalmente, se busca que los estudiantes desarrollen textos de mayor complejidad y de géneros diversos, orales y escritos.

5. Tain doy nütramkayal ('Para conversar más'): se despliega en las dos páginas siguientes, las últimas de cada lección. Incluye un diálogo de extensión superior a los anteriores, que reúne contenidos de las situaciones comunicativas ya revisadas. A lo largo de los diálogos de todas las secciones Taiñ doy nütramkayal del método, se desarrolla una historia que concluye en la última lección. Además de este diálogo, se presentan actividades de ejercitación, un resumen de frases útiles, cuadros de contenidos fonológicos (uno orientado a los fonemas y otro a la entonación), y textos temáticos acerca de la cultura mapuche. El objetivo de esta sección es presentar una síntesis de los contenidos y promover la aplicación de ellos en una situación comunicativa real. 


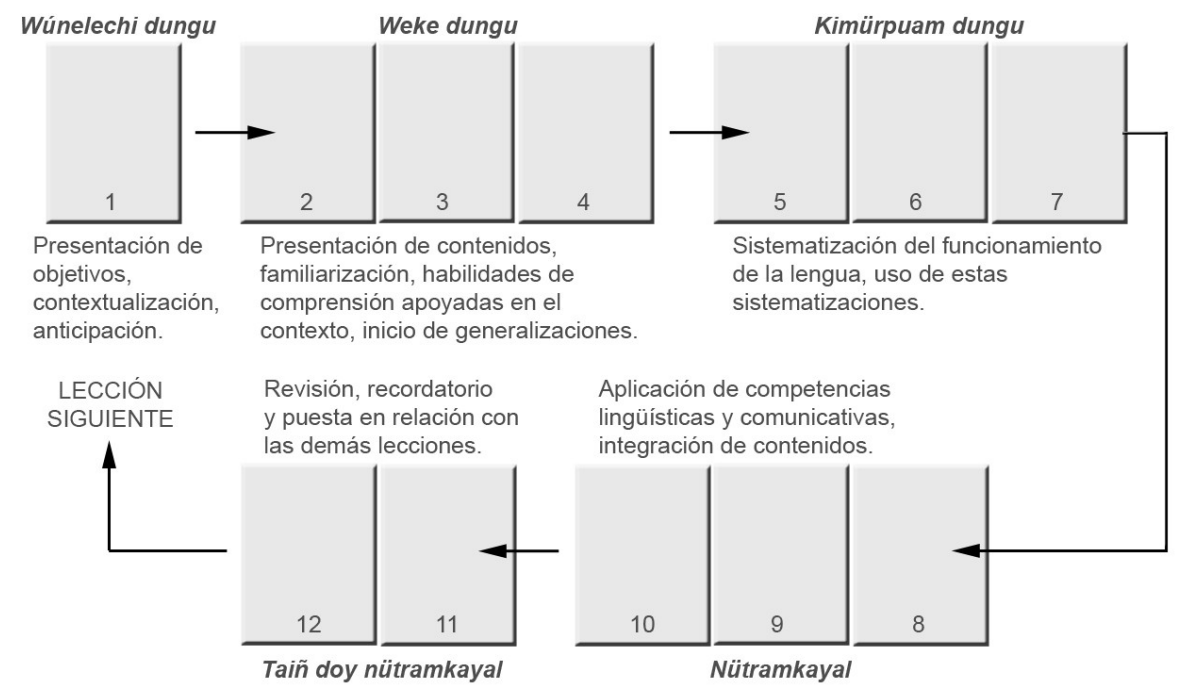

Figura 1: Esquema de organización local de cada lección

En la Figura 1 se presenta un esquema de la organización local de cada lección. En él, cada recuadro representa una página (que señala su orden correlativo mediante un número). En los márgenes superior e inferior, en cursivas, se presenta el nombre de cada sección. Al centro, en fuente regular, se presentan los objetivos didácticos de cada sección.

\section{CONCLUSIONES}

De acuerdo con lo presentado, el método de enseñanza-aprendizaje Folil Mapudungun es multidimensional en un doble sentido: no solo organiza sus objetivos y contenidos de acuerdo con una malla de criterios paralelos, de naturaleza tanto funcional como formal (por ejemplo, situaciones, funciones, estructuras, etc.), sino que incluye dichos criterios organizativos en una arquitectura de diseño mayor. Este considera aspectos diversos del uso de la lengua (principios discursivos, puesta en práctica de la lengua en situaciones reales), de su relación con la cultura (contextualización y relación del conocimiento cultural con los conocimientos lingüísticos), y de los principios didácticos que pueden orientar y allanar el proceso de adquisición de una segunda lengua.

Creemos que esta organización permite tender a la superación de la dicotomía entre contenidos y metodología - entre producto y proceso-, a la vez que favorecer el desarrollo de los diversos aspectos que conforman la competencia comunicativa. Siguiendo los desarrollos sociolingüísticos y aplicados mencionados, el plan de presentación de la lengua considera la construcción de la competencia lingüística a la 
par de la sociolingüística (tanto sociocultural y discursiva), y ambas en relación con la competencia estratégica para la acción y la resolución de problemas.

Finalmente, sostenemos el aporte de este método en el panorama actual de efervescencia de los movimientos de recuperación lingüística de los pueblos originarios, y en particular del pueblo mapuche. En este contexto, este método pone a disposición un programa y un material útil para los procesos de enseñanza-aprendizaje que hoy son desarrollados por actores sociales diversos. La orientación del método y la organización de su programa debieran favorecer los logros pedagógicos en la adquisición inicial de la lengua mapuche, arista aún no resuelta. Por último, la constatación de estos aportes no puede llevar al olvido de la interrelación de todo proceso educativo con los contextos socioculturales en que se desenvuelve. En este sentido, esta propuesta se enmarca en un horizonte más amplio, sujeto a constante revisión y profundización por parte de diversos actores, entre estos está, por cierto, la academia.

$$
\begin{array}{r}
\text { Universidad de Concepción* } \\
\text { Facultad de Ciencias Sociales } \\
\text { Barrio Universitario s/n. Casilla 160 C, Correo 3. Concepción (Chile) } \\
\text { robecerr@udec.cl }
\end{array}
$$

El autor agradece el apoyo recibido del proyecto Fondo del Libro de Investigación 2014, folio 55777.

\section{OBRAS CITADAS}

Adam, Jean-Michel (1992). Los textos: Tipos y prototipos. Relato, descripción, argumentación, explicación, diálogo. París: Natham.

Alonqueo, Martín (1987). El habla de mi tierra. Temuco: Impresos Kolping.

Antileo, Antonio (1976). Texto mapuche para hispanohablantes: Método audiolingual. Santiago: Universidad Católica de Chile.

Anthony, Edward (1963). "Approach, method, and technique”. English Language Teaching 17: 63-67.

Bartolomé, Miguel (2006). Procesos interculturales: Antropología política del pluralismo cultural en América Latina. México: Siglo XXI.

Bengoa, José (2007). La emergencia indígena en América Latina. Santiago: Fondo de Cultura Económica.

Boccara, Guillaume (2012). "La interculturalidad como campo social". Cuadernos Interculturales 18: 11-30.

(2002). "Colonización, resistencia y etnogénesis en las fronteras americanas". Colonización, resistencia y mestizaje en las Américas (Siglos XVI-XX), ed. Guillaume Boccara. Lima/Quito: IFEA / Abya Yala: 47-82.

Brown, H. Douglas (2001). Teaching by Principles: An Interactive Approach to Language Pedagogy. Nueva York: Longman. 
Calsamiglia, Helena y Amparo Tusón (2007). Las cosas del decir. Manual de análisis del discurso. Barcelona: Ariel.

Canale, Michael y Merril Swain (1980). "Theoretical bases of communicative approaches to second language learning and testing". Applied Linguistics 1, 1: 1-47.

Cañulef, Eliseo (1998). Introducción a la Educación Intercultural Bilingüe en Chile. Temuco: Instituto de Estudios Indígenas.

Catrileo, María (2010). La lengua mapuche en el siglo XXI. Valdivia: Universidad Austral de Chile.

— Mapudunguyu: Curso de lengua mapuche. Valdivia: Universidad Austral de Chile, 1987.

Chiodi, Francesco y Elisa Loncón (1995). Por una nueva política del lenguaje. Temas y estrategias del desarrollo lingüistico del mapudungun. Temuco: Pehuén / Universidad de La Frontera.

Conferencia Mundial de Derechos Lingüísticos (1996). Declaración Universal de los Derechos Lingüísticos. Disponible en:

$\mathrm{http} / / / \mathrm{www}$. unesco.org/cpp/sp/declaraciones/linguisticos.htm

Consejo de Europa (2002). Marco común europeo de referencia para las lenguas: Aprendizaje, enseñanza, evaluación. Madrid: Ministerio de Educación, Cultura y Deporte / Anaya.

Da Silva, Tomaz Tadeu (2001). Espacios de identidad. Nuevas visiones sobre el currículum. Barcelona: Octaedro.

De Moesbach, Ernesto Wilhelm (1963). Idioma Mapuche. Padre Las Casas, Temuco: San Francisco.

Donoso, Andrés (2010). Educación y nación al sur de la Frontera. Organizaciones mapuche en el umbral de nuestra contemporaneidad, 1880-1930. Santiago: Pehuén.

Duranti, Alessandro (2003). "Language as Culture in U.S. Anthropology; Three Paradigms". Current Anthropology 44, 3: 323-347.

García, Álvaro (2000). Cómo se diseña un curso de lengua extranjera. Madrid: Arco Libros.

Golluscio, Lucía (2006). El pueblo mapuche: poéticas de pertenencia y devenir. Buenos Aires: Biblos.

— (Comp.) (2002). Etnografia del Habla: Textos fundacionales. Buenos Aires: Eudeba.

Gumperz, John (2002). "Las bases lingüísticas de la competencia comunicativa". Etnografia del Habla: Textos fundacionales, comp. Lucía Golluscio. Buenos Aires: Eudeba: 151-163.

Gundermann, Hans; Canihuan, Jaqueline; Clavería, Alejandro y César Faúndez (2011). "El mapuzugun, una lengua en retroceso". Atenea 503: 111-131.

Halliday, Michael (2005). El lenguaje como semiótica social: La interpretación social del lenguaje y del significado. México: Fondo de Cultura Económica.

Hasler, Felipe; Mariano, Héctor y Andrea Salazar (2011). "La enseñanza de mapudungun en la actualidad: Breve guía bibliográfica de materiales de 
Folil Mapudungun: organización de un método con enfoque comunicativo

enseñanza de la lengua mapuche". Educación intercultural bilingüe en América Latina y el Caribe: balances, desafios y perspectivas, ed. Elisa Loncón y Ana Hecht. Santiago: Fundación Equitas: 192-202.

Huenchullán, Carolina (2007). "La institucionalización de la educación intercultural bilingüe en Chile: Contexto, situación actual y desafíos". Patrimonio Cultural Mapunche. Derechos culturales y patrimonio educacional mapunche, ed. Teresa Durán, Desiderio Catriquir y Arturo Hernández. Temuco: Universidad Católica de Temuco: 207-226.

Hymes, Dell (1972). "On communicative competence". Sociolinguistics. Selected Readings, ed. John B. Pride y Janet Holmes. Harmondsworth, Inglaterra: Penguin: 269-293.

Johnson, Keith (2008). Aprender y enseñar lenguas extranjeras. Una introducción. México: Fondo de Cultura Económica.

Littlemore, Jeannette (2011). Applying cognitive linguistics to second language learning and teaching. Basingstoke, Inglaterra: Palgrave Macmillan.

López, Luis Enrique (2007). "Diversidad cultural, multilingüismo y reinvención de la educación intercultural bilingüe en América Latina". Patrimonio Cultural Mapunche. Derechos culturales y patrimonio educacional mapunche, ed. Teresa Durán, Desiderio Catriquir y Arturo Hernández. Temuco: Universidad Católica de Temuco: 339-363.

Ministerio de Educación (2013a). Kimelkawe ñi chillkangeal mapuzugun. Lengua Mapuche/Texto de Estudio. Santiago de Chile: Ministerio de Educación.

— (2013b). Chew ñi rekuluwam ta ti pu kimeltuchefe mapuzugun. Guía del educador (a) tradicional. Santiago, Chile: Ministerio de Educación.

—_ (2011a). Programa de estudio de Primer Año Básico Sector Lengua Indígena Mapuzugun. Santiago, Chile: Ministerio de Educación.

_ (2011b). Programa de estudio de Segundo Año Básico Sector Lengua Indígena Mapuzugun. Santiago, Chile: Ministerio de Educación.

— (2011c). PEIB-Orígenes. Estudio sobre la implementación de la Educación Intercultural Bilingüe. Santiago, Chile: Ministerio de Educación.

Norin, Wenceslao; Becerra, Rodrigo; Mellico, Fresia; Fajardo, Juan; Norin, Fresia; Huentemil, Julio; Huaiquillán, Octavio; Marileo, Armando y Eliseo Huencho (2013). Folil Mapudungun 1. Chumngechi tañi kimeltungeken $k a$ chillkatungeken mapudungun. Método de enseñanza-aprendizaje de la lengua mapuche. Concepción: Trama Impresores.

Pozo, Gabriel (2014). "¿Cómo descolonizar el saber? El problema del concepto de interculturalidad. Reflexiones para el caso mapuche". Polis 38: 2-13.

Raguileo, Anselmo (1990). Educación bilingüe e intercultural y el alfabeto mapuche. Inédito. Red por los Derechos Educativos y Lingüísticos de los Pueblos Indígenas de Chile [Red DELPICH]. (2011). Resoluciones del II Congreso de las Lenguas Indigenas de Chile. Disponible en http://www.archivochile.com/carril_c/cc2012/cc2012-017.pdf 
Richards, Jack y Theodore Rodgers (1982). "Method: approach, design, procedure". TESOL Quarterly 16, 2: 153-68.

Searle, John R. (1975). "A taxonomy of illocutionary acts". Language, Mind, and Knowledge, (Minneapolis Studies in the Philosophy of Science, vol. 7), ed. Keith Günderson. Minneapolis, EE.UU.: University of Minneapolis Press: 344-369.

Treviño, Ernesto; Donoso, Francisca; Aguirre, Elisa; Fraser, Pablo; Godoy, Felipe; Inostroza, David; Chávez, Bárbara; Place, Katherine; Tapia, Paula y Paula Castro (2012). Educación para preservar nuestra identidad cultural: Desafios de implementación del sector de Lengua Indígena en Chile. Santiago, Chile: Ministerio de Educación.

Universidad Tecnológica Metropolitana y Corporación Nacional de Desarrollo Indígena (2008). Perfil sociolingüistico de comunidades mapuche de la VIII, IXy $X$ Región. Informe de resultados 2008. Santiago: Universidad Tecnológica Metropolitana.

Wiley, Terrence (2014). "The problem of defining Heritage and Community Languages and theirs speakers. On the utility and limitations of definitional constructs". Handbook of Heritage, Community, and Native American Languages in the United States. Research, policy and educational practice, ed. Terrence Wiley; Joy K. Peyton; Donna Christian; Sarah C. K. Moore y Na Liu. Nueva York: Routledge: 19-26.

Wilkins, David (1972). An investigation into the linguistic and situational common core in a Unit/Credit System. Estrasburgo: Consejo de Europa. Disponible en http://files.eric.ed.gov/fulltext/ED082545.pdf

Wittig, Fernando (2009). "Desplazamiento y vigencia del mapudungun en Chile: Un análisis desde el discurso reflexivo de los hablantes urbanos". RLA 47, 2: 135-155.

Zabalza, Miguel (2009). Diseño y desarrollo curricular. Madrid: Narcea. 